To the Editors:

\title{
Multiple autoimmune phenomena preceding a lymphoproliferative disorder
}

Karunatilake and Pidcock [1] recently described an interesting patient with autoimmune manifestations, cytopenias and lymphoproliferation. However, the authors did not comment on several important aspects. Did the patient have any lymphadenopathy, and was there a family history of a similar condition? Was the presence of double negative $T$ cells in the peripheral blood looked for, was an aptotosis assay done, and if abnormal, were relevant Fas pathway gene defects looked for? The patient described may in fact have had the autoimmune lymphoproliferative syndrome (ALPS).

ALPS is an inherited disorder of lymphocyte apoptosis associated with autoimmune cytopenias and lymphoproliferation. These patients have an increased risk of lymphoma. It is the first human disease whose aetiology has been attributed to a primary defect in apoptosis, ie programmed cell death (PCD). Most cases are associated with heterozygous mutations in the Fas (TNFRSF6) gene. Mutations in Fas ligand, caspase- 8 and caspase- 10 have been found in some patients.

With increasing awareness, adults with ALPS are now being diagnosed more frequently [2]. Its detection helps plan appropriate management and allows family studies to be undertaken with a view to genetic counselling.

In 1967, Canale and Smith [3] first described five patients with autoimmune cytopenias, splenomegaly and lymphadenopathy. Sneller, et al [4] found increased numbers of double negative $T$ cells in two other patients with a similar presentation. They also proposed that these patients might be the human equivalent of a murine disease caused by lpr and gld mutations. In 1992, lpr mice were found to have defects in their Fas gene [5]. Thereafter, Fas mutations were found in patients with ALPS [6]. Some patients first described by Canale and Smith, were also found to have Fas gene defects.
Recognition of similarities between ALPS patients and mouse models (lpr and gld mice) allowed scientists to demonstrate Fas gene mutations in patients with this condition. This illustrates a situation where mouse genetics specifically directed scientists to finding the cause of a human disease. Finding defects in the Fas apoptotic pathway in some ALPS patients also helped to study this physiologically important pathway in detail. It has given us an unique insight into the control of programmed cell death, lymphocyte homeostasis and the termination of an immune response. This in turn has added to our understanding of malignancy, and apoptosis, and has had implications for targeted drug design.

\section{References}

1. Karunatilake H, Pidcock ME. Multiple autoimmune phenomena preceding a lymphoproliferative disorder. Ceylon Medical Journal 2007; 52: 113.

2. Deutsch M, Tsopanou E, Dourakis SP. The autoimmune lymphoproliferative syndrome (Canale-Smith) in adulthood. Clinical Rheumatology 2004; 23: 43-4.

3. Canale VC, Smith CH. Chronic lymphadenopathy simulating malignant lymphoma. Journal of Paediatrics 1967; 70: 891-9.

4. Sneller MC, Straus SE, Jaffe ES, Jaffe JS, Fleisher TA, et al. A novel lympho-proliferative/autoimmune syndrome resembling murine $1 \mathrm{pr} / \mathrm{gld}$ disease. Journal of Clinical Investigation 1992; 90: 334-41.

5. Watanabe-Fukunaga R, Brannan CI, Copeland NG, Jenkins NA, Nagata S. Lymphoproliferation disorder in mice explained by defects in Fas antigen that mediates apoptosis. Nature 1992; 356: 314-7.

6. Fisher GH, Rosenberg FJ, Straus SE, Dale JK, Middleton LA, et al. Dominant interfering Fas gene mutations impair apoptosis in a human autoimmune lymphoproliferative syndrome. Cell 1995; 81: 935-46.

Suranjith L Seneviratne, St Mary's Hospital and Imperial College London, United Kingdom. E-mail <Suranjith.Seneviratne@imperial.nhs.uk>. 